\title{
Atomic-Scale Mass Sensing Using Carbon Nanotube Resonators
}

\author{
Hsin-Ying Chiu, ${ }^{\dagger}$ Peter Hung, ${ }^{\dagger}$ Henk W. Ch. Postma, ${ }^{\ddagger}$ and Marc Bockrath ${ }^{\star}, \dagger$ \\ Department of Applied Physics, California Institute of Technology, Pasadena, \\ California 91125, and Department of Physics and Astronomy, California State \\ University, Northridge, Northridge, California 91330
}

Received July 19, 2008; Revised Manuscript Received September 25, 2008

\begin{abstract}
Ultraminiaturized mass spectrometers are highly sought-after tools, with numerous applications in areas such as environmental protection, exploration, and drug development. We realize atomic scale mass sensing using doubly clamped suspended carbon nanotube nanomechanical resonators, in which their single-electron transistor properties allows self-detection of the nanotube vibration. We use the detection of shifts in the resonance frequency of the nanotubes to sense and determine the inertial mass of atoms as well as the mass of the nanotube. This highly sensitive mass detection capability may eventually enable applications such as on-chip detection, analysis, and identification of compounds.
\end{abstract}

Because of their small size, nanoscale systems are highly sensitive to their environment, affording great potential for sensing applications. Chemical and biological charge-based sensors using carbon nanotubes ${ }^{1,2}$ and nanowires ${ }^{3}$ have already been demonstrated. Nanoelectromechanical systems have been proposed for highly sensitive mass detection of neutral species,, 45 and significant progress has been made in using nanofabricated resonators ${ }^{6-8}$ and carbon nanotubes ${ }^{9-12}$ for mass sensing. However, the ultimate limit of atomic resolution mass sensitivity remains unrealized. Here we demonstrate that individual double-clamped single-walled carbon nanotube resonators are capable of atomic-scale mass sensing and determining the inertial mass of atomic species. Analysis of our data also yields the nanotube mass, giving a general approach to determining the mass of molecular nanostructures. Our results open the door to ultraminiaturized mass spectroscopy or arrays, potentially enabling on-chip detection and identification of unknown analytes, as well as the study of single atom adsorption and desorption.

We fabricate our devices by first growing nanotubes from catalyst islands ${ }^{13}$ under either pure $\mathrm{CH}_{4}{ }^{13}$ or a $\mathrm{CH}_{4} / \mathrm{H}_{2}$ mixture ${ }^{14}$ on an oxidized $\mathrm{Si}$ wafer. We then attach $\mathrm{Pd} / \mathrm{Au}$ source/drain electrodes and a side gate using electron beam lithography (EBL). In a second step, a layer of poly(methyl methacrylate) (PMMA) is spun on the chip, and a $\sim 450 \mathrm{~nm}$ window is opened over a segment of the nanotube between the electrodes, again using EBL. The $\mathrm{SiO}_{2}$ is etched using buffered HF to suspend the carbon nanotube within the window. Following a critical point drying step, the samples

* To whom correspondence should be addressed. E-mail: mwb@ caltech.edu.

$\dagger$ California Institute of Technology.

$¥$ California State University Northridge. are electrically tested at room temperature; we then load selected devices into a custom-built cryostat, which is maintained at a high vacuum. Figure 1a shows a scanning electron microscope (SEM) image of a completed suspended nanotube transistor device.

At the temperature $T \approx 6 \mathrm{~K}$ of our experiment, the nanotubes act as single-electron transistors (SET) in which the charge on the nanotube is an integer multiple of the electron charge $e .{ }^{15}$ As the gate voltage $V_{\mathrm{g}}$ is swept, electrons enter the SET one at a time, with each transition between discrete charge states producing a Coulomb peak in the source-drain conductance (see e.g. ref 16). Figure 1b shows this behavior. To measure the resonant vibrational amplitude of the carbon nanotubes, we drive their motion electrostatically and take advantage of these SET properties to sense the resulting displacement capacitively. ${ }^{17}$ This approach is adapted from that described by Sazonova et al. ${ }^{18}$ Figure 1c shows a diagram of the experimental setup. A voltage $V_{\mathrm{sd}}=$ $U_{\text {sd }}+V_{0} \cos \omega t$ is applied across the source and drain electrodes, where $t$ is the time and $\omega$ is the frequency, while a voltage with a detuned $\mathrm{AC}$ component at frequency $\omega+$ $\delta \omega$ is applied to the gate so that $V_{\mathrm{g}}=V_{\mathrm{DC}}+V_{\mathrm{AC}} \cos [(\omega+$ $\delta \omega) t]$. The nanotube transistor mixes the signals to yield a current $I$ that is measured by a lock-in amplifier with a $\sim 1$ $\mathrm{Hz}$ bandwidth at the difference frequency $\delta \omega$. Tuning $\omega$ to a nanomechanical resonance of the nanotube produces vibrations that modulate the tube-gate capacitance, changing the measured $I$ in proportion to the vibration amplitude.

Figure 2a shows a color-scale plot of $I$ versus $V_{\mathrm{DC}}$ and $f$ $=\omega / 2 \pi$ at $T \approx 6 \mathrm{~K}$ for a suspended nanotube device. The tube diameter $d$ is $\sim 1 \mathrm{~nm}$, as determined by atomic force microscopy on the substrate-supported segment. From $d$, we 
(a)

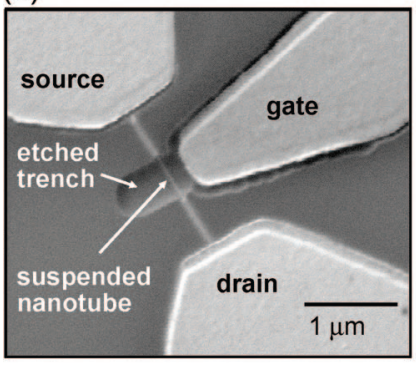

(b)

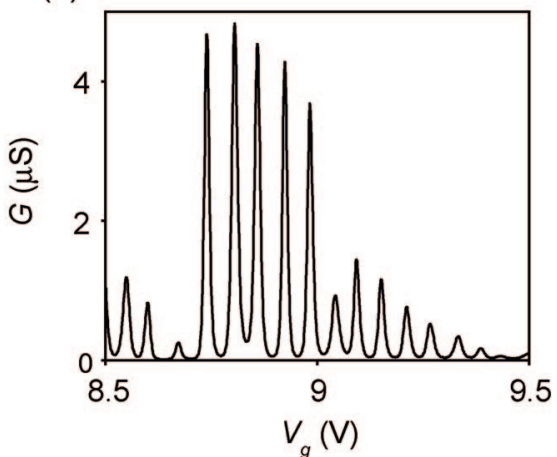

(c)

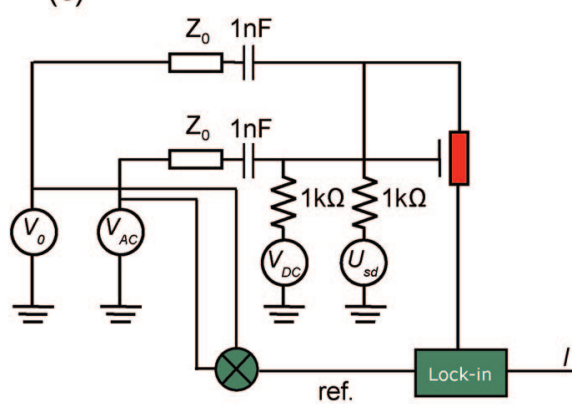

Figure 1. Device geometry of suspended carbon nanotubes, single-electron transistor characteristics, and high-frequency measurement setup. (a) Scanning electron microscope image of a representative suspended nanotube device with source, drain, and gate electrodes. (b) Source-drain conductance versus gate voltage showing Coulomb peaks and single-electron transistor behavior at $T \approx 6 \mathrm{~K}$. (c) Schematic diagram of the measurement circuit. The transmission lines with characteristic impedance $Z_{0}=50 \Omega$ are both terminated at each end with a $50 \Omega$ resistor to ground. The voltages $V_{0}, V_{\mathrm{AC}}, V_{\mathrm{DC}}$, and $U_{\mathrm{sd}}$ are described in the text. The nanotube is shown in red.
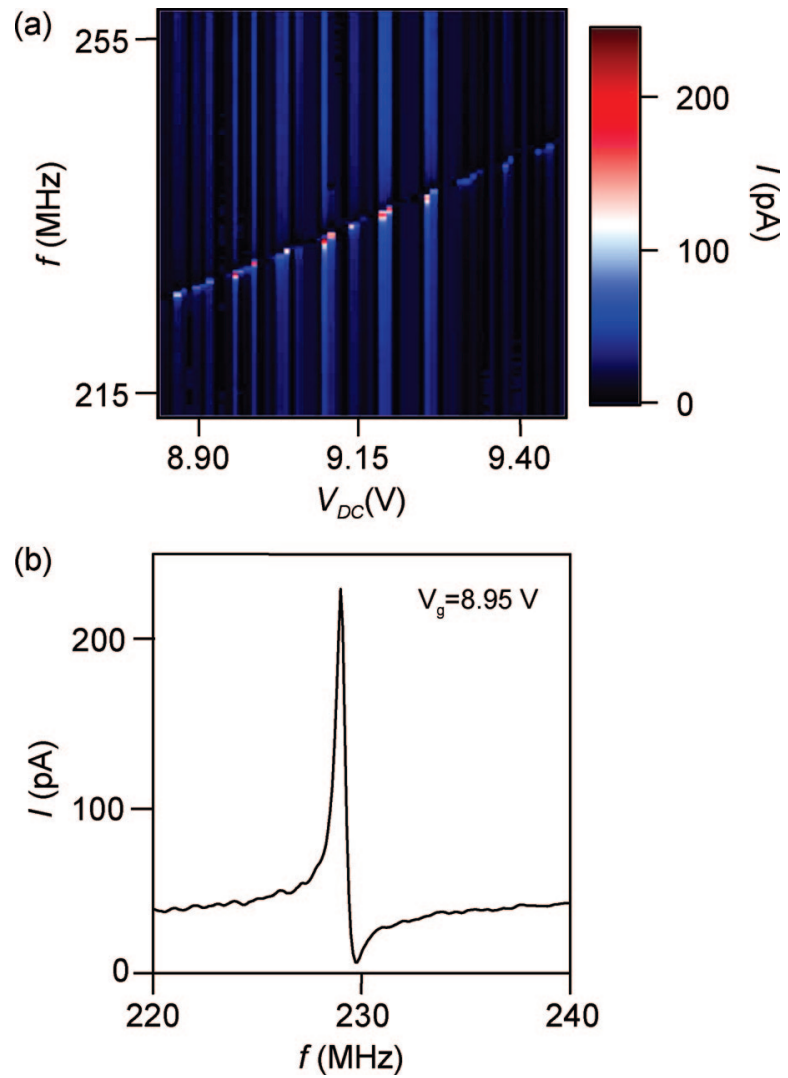

Figure 2. Nanotube resonator vibration characteristics. (a) Color plot of the mixing current $I$ detected by the lock-in amplifier at the difference frequency, as described in the text, versus DC gate voltage $V_{\mathrm{DC}}$ and drive frequency. (b) Line trace of the mixing current $I$ versus the drive frequency $f$. A peak occurs when the frequency is tuned to a nanomechanical resonance of the nanotube device. Achieving such large peak heights and resulting signal-tonoise ratio required the side gate to be in close proximity to the nanotube, typically $150 \mathrm{~nm}$ or less.

estimate the suspended tube mass $m_{0} \sim 1000 \mathrm{zg}$. Typical values for the applied voltages are $V_{0} \approx 0.8 \mathrm{mV}$ and $V_{\mathrm{AC}} \approx$ $0.8 \mathrm{mV}$. The current signal measured at the lock-in amplifier is proportional to $\mathrm{d} G / \mathrm{d} V_{\mathrm{g}}{ }^{18,19}$ On the shoulders of the Coulomb peaks $\mathrm{d} G / \mathrm{d} V_{\mathrm{g}}$ becomes large, as high as $\sim 380 \mu \mathrm{S} /$ $\mathrm{V}$, producing the vertical stripelike background in Figure 2a.
The approximately horizontal feature corresponds to a nanomechanical resonance. Increasing $V_{\mathrm{DC}}$ increases the tension in the nanotube, raising its resonance frequency. ${ }^{18,20}$ This produces a finite-slope feature. ${ }^{18,19}$ From the small slack we observe in our SEM images, we expect that the nanotube in our device behaves as a stretched string. ${ }^{18,21}$ Following a similar analysis as given in ref 18 , we find the characteristic vibration amplitude for our system with the given $V_{0}$ and $V_{\mathrm{AC}}$ is $\sim 1 \mathrm{~nm}$. A line trace of $I$ versus $f$ is shown in Figure $2 \mathrm{~b}$, showing that the resonance has a quality factor $Q \sim 200$. The characteristic fundamental-mode resonance frequencies $f_{0}=100-300 \mathrm{MHz}$ are consistent with those expected for single-walled nanotubes with the $\sim 400-500 \mathrm{~nm}$ length and nanometer-scale diameters under study. ${ }^{18,22}$ Note that the line shape is asymmetric, which may indicate the onset of nonlinearity. ${ }^{18}$ At sufficiently large drives, such nonlinearity may produce an upper bound on the signal-to-noise ratio for frequency measurements, ${ }^{23}$ although this is not a limitation of our present experiment.

After identifying a promising resonance peak (typically $>100 \mathrm{pA}$ for the given $V_{\mathrm{AC}}$ and $V_{0}$ ), we expose the device to an atomic or molecular beam. The inset to Figure 3 shows the experimental setup, which is similar to that employed by Yang et al. ${ }^{7}$ to mass load top-down fabricated nanowire mass sensors. A reservoir maintained near room temperature connects to the sample space through a small aperture $(\approx 135$ $\mu \mathrm{m}$ in diameter) and is filled with the analyte gas. Baffles maintained near liquid helium temperature are interposed between the aperture and sample and serve to block any atoms that are not on a ballistic trajectory from the inlet. Assuming that the gas effuses into the sample space through the aperture enables the determination of the atomic flux $\phi_{0}$ through the aperture, yielding $\phi_{0}=P /\left(2 \pi m k_{\mathrm{B}} T\right)^{1 / 2}$, where $P$ is the pressure in the reservoir, $m$ is the atomic or molecular mass, $k_{\mathrm{B}}$ is Boltzmann's constant, and $T$ is the temperature. From $\phi_{0}$ and the distance between the aperture and the sample $h \approx 0.90 \mathrm{~m}$, we determine the atomic flux arriving at the nanotube, $\phi_{\mathrm{n}}=A \phi_{0} / 2 \pi h^{2}$, where $A \approx 1.4 \times 10^{-8} \mathrm{~m}^{2}$ is the area of the aperture used in our experiments. 


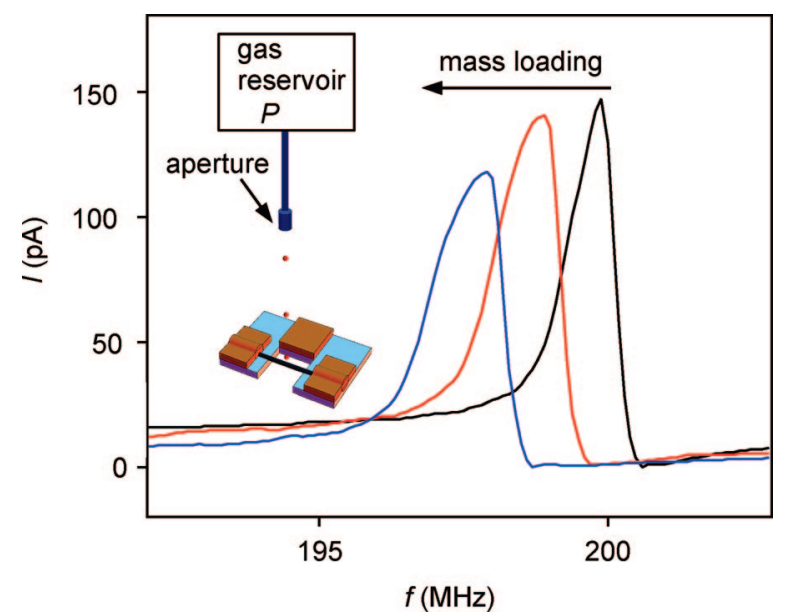

Figure 3. Response of nanomechanical resonance under mass loading and experimental setup. Main panel: mixing current $I$ versus drive frequency $f$ for a resonator device during mass loading by $\mathrm{Xe}$. The black curve shows the initial resonance, the red curve after mass loading for $\approx 600 \mathrm{~s}$, and the blue curve after mass loading for $\approx 1600$ s. Inset: schematic diagram of the experimental setup for mass loading nanotube resonator devices. Gas atoms or molecules maintained near room temperature effuse through $\mathrm{a} \approx 135$ $\mu \mathrm{m}$ diameter aperture and travel ballistically along the line of sight to the nanotube.

We first fill the reservoir with Xe. The main panel of Figure 3 shows that the resonance traces shift and broaden as the atoms land on the nanotube and adsorb on the surface. The amplitude also decreases slightly. The broadening and amplitude decrease indicate a reduction in the effective quality factor of the nanotube resonator, although further experiments are required to address the origin of this behavior. The black trace shows the initial resonance, while the red and blue traces show the resonance after $\approx 600 \mathrm{~s}$ and $\approx 1600 \mathrm{~s}$, respectively. Based on the resonance frequency shift $\Delta f_{0} \approx 3 \mathrm{MHz}$, which in a harmonic oscillator picture for the vibrating nanotube should follow $\Delta f_{0} / f_{0} \cong m_{\mathrm{A}} / 2 m_{0}$, where $m_{\mathrm{A}}$ is the adsorbed mass, we deduce that the $m_{A}$ corresponds to approximately $100-200 \mathrm{Xe}$ atoms. At the low temperature of our experiment, desorption does not readily occur and the resonance frequency remains stable once the reservoir is pumped out. Finally, we note that in an independent trial we mass loaded the resonator with a constant background pressure of $\mathrm{He}$ and found that the frequency increased with the gate voltage magnitude, with a similar slope as in vacuum.

The large observed shifts for the small adsorbed mass suggests our devices could be sensitive to the arrival of individual atoms. To explore this, we fixed the drive frequency to the side of the resonance peak and then monitored the lock-in current as a function of time. Changes in the resonance frequency thereby produce changes in the lock-in current. We then expose the nanotube to an atomic arrival rate of $\sim 3$ atoms/s by filling the reservoir to a pressure of $\sim 1$ Torr, as shown in Figure 4a. Before the mass loading begins by filling the reservoir, $I$ is roughly constant, demonstrating the stability of our device. Once the Xe fills the reservoir, at the time indicated by the arrow, $I$ begins to increase as the resonance peak shifts downward in frequency
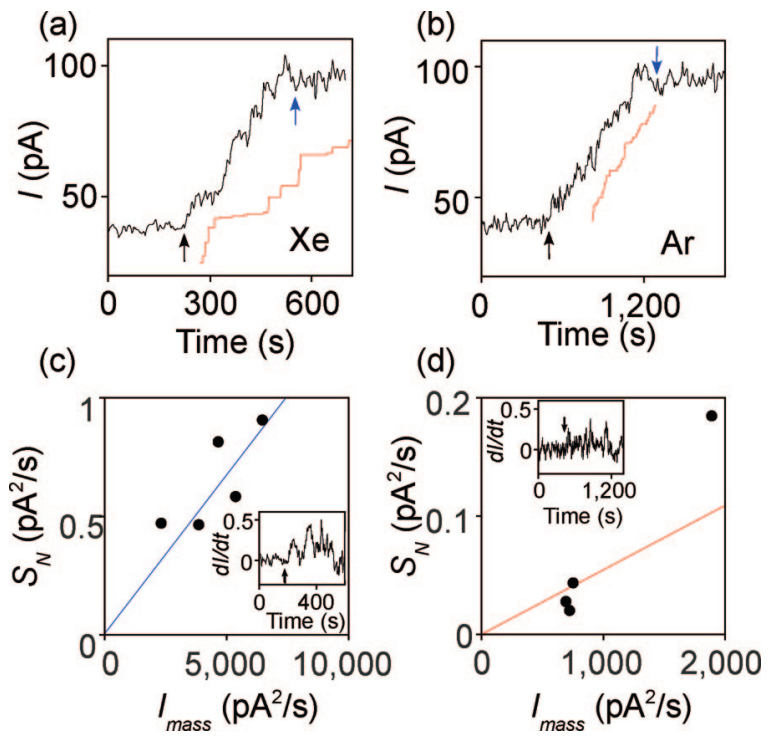

Figure 4. (a) Main panel, black curve: mixing current $I$ versus time as the nanotube resonator is mass loaded with Xe. The mass loading begins at the time indicated by the black arrow and ends at the blue arrow. Main panel, red curve: a simulated data trace with the parameters given in the text. Inset: time derivative of the current smoothed with a $50 \mathrm{~s}$ window averaging. The black arrow indicates the start of the mass loading, and the blue arrow the end. (b) Similar data as in (a), but for Ar. (c) The effective shot noise power versus effective mass current for a number of different data runs for Xe and (d) for Ar. In measuring the shot noise, care was taken to select only those data sets in which the current was generally monotonically increasing. Some data sets, particularly after the nanotubes had a significant amount of accumulated mass, showed large up and down current fluctuations relative to the initially measured background noise, which we attribute to the surface diffusion of adsorbed atoms, possibly driven by the impinging atoms. Data sets with such fluctuations did not generally yield consistent results for the shot noise versus the adsorbate atomic mass.

due to the mass loading. Remarkably, $I$ shows a series of steplike features with the largest $\sim 10 \mathrm{pA}$ as the Xe flux is maintained. After $\sim 600 \mathrm{~s}$, the reservoir is emptied and the current rise stops.

To understand the origin of these features, we note that the expected shift in frequency $\Delta f_{0}$ depends on the location at which the atom adsorbs, with the maximum effect occurring for adsorption in the center of the nanotube. Modeling the nanotube as a stretched string and using the result for the frequency shift due to an attached point mass (e.g., ref 24), we find that for $m \ll m_{0}$, where $m$ is the atomic mass

$$
\frac{\Delta f_{0}}{f_{0}} \cong-\frac{m}{m_{0}} \sin (\pi a / L) \sin [\pi(L-a) / L]
$$

where $a$ is the position along the nanotube at which the atom adsorbs and $L$ is the suspended length. Assuming the atoms land anywhere along the nanotube with equal probability, the mean value of $\Delta f_{0} / f_{0}$ is $\left\langle\Delta f_{0} / f_{0}\right\rangle=m / 2 m_{0}$, and the mean square value $\left\langle\left(\Delta f_{0} / f_{0}\right)^{2}\right\rangle=3 / 8\left(\mathrm{~m} / \mathrm{m}_{0}\right)^{2}$. Here we assume that we are exciting the fundamental mode, although relaxing that assumption yields the same results.

The red trace in Figure 4a shows a simulated time trace of the current based on eq 1 , using the parameters $m_{0}=$ $1000 \mathrm{zg}, m=m_{\mathrm{Xe}}=0.218 \mathrm{zg}$, and an adsorption rate of 
0.07 atoms/s, determined from $m_{0}$ and the slope of the frequency versus time curve. The trace, plotted without free parameters, shows a series of steps each corresponding to the adsorption of an atom. The largest single steps occur when the atoms land near or at the center of the tube and are of magnitude $5.8 \mathrm{pA}$. In comparison, the root-meansquare noise level we observe is $\sim 1.4 \mathrm{pA}$. Based on the relations found in ref 25 and the physical parameters of our devices, the noise is too large to be accounted for by thermomechanical or temperature fluctuation noise. We therefore attribute this background noise to environmental charge fluctuations, which would yield a charge noise of $\sim 10^{-4} \mathrm{e} / \mathrm{Hz}^{1 / 2}$, similar to that found in previous experiments on nanotube SETs (e.g., ref 26). This calculation suggests that our measurement is sensitive to the added mass of individual Xe atoms provided they land near the tube center. Steps of this order of magnitude are apparent in Figure 4a, although we cannot exclude the possibility of several atoms adsorbing on the nanotube within the $1 \mathrm{~s}$ measurement time constant. On the basis of $\left\langle\Delta f_{0} / f_{0}\right\rangle$ given above and the total $\Delta f_{0} / f_{0} \sim 3600 \mathrm{ppm}$ for the entire mass loading period, we estimate the nanotube captured $\sim 30 \mathrm{Xe}$ atoms during the mass loading process. This indicates that only about $3 \%$ of the incident $\mathrm{Xe}$ atoms stick to the surface upon colliding with the nanotube, consistent with molecular dynamic calculations of $\mathrm{Xe}$ collisions with nanotubes, ${ }^{27,28}$ which predict that nanotubes will capture and adsorb only a small fraction of incident atoms. Note that, compared to graphene, the highly curved geometry of a nanotube produces a range of effective incident angles to the normal and is expected to somewhat lower the binding potential energy. In addition, the sticking coefficient may be further reduced by the presence of surface impurities, ${ }^{29}$ e.g., processing residue.

Figure $4 \mathrm{~b}$ shows similar data taken by mass loading the nanotube with Ar. The reservoir is loaded at the time indicated by the arrow and emptied after about $\sim 1200$ s. The mean mass accumulation rate is $\sim 2$ times smaller than for the data shown in Figure $4 \mathrm{a}$, although the reservoir pressure was larger, suggesting that the sticking coefficient for Ar on the nanotube is smaller than that for Xe. The red curve below the data shows a simulated trace with $m_{0}=$ $1000 \mathrm{zg}, m=m_{\mathrm{Ar}}=0.066 \mathrm{zg}$, and an adsorption rate of $\sim 0.09$ atoms/s, which appears similar to the data. Compared to the data for $\mathrm{Xe}$, the Ar data has narrower plateaus and shorter steps.

To use nanotubes as an atomic scale mass sensor, we require a measurement of $m_{0}$. To achieve this, we note that these frequency shifts during the adsorption could be viewed as arising from shot noise due to the arriving atoms, similar to the adsorption-desorption noise expected for a resonator immersed in a gas. ${ }^{25,30,31}$ Studying the shot noise enables the statistical fluctuations in mass changes due to atomic arrival to be analyzed even when the smallest individual steps may be obscured by the noise floor present in the measurement. We now compute the expected magnitude of the shot noise. Shot noise produces fluctuations in the mass arrival current $\mathrm{d} m_{\mathrm{A}} / \mathrm{d} t$. The mean value of $\mathrm{d} m_{\mathrm{A}} / \mathrm{d} t=\Gamma m$, where $\Gamma$ is the mean atomic adsorption rate. This is related to the mean rate of increase $H=\mathrm{d} I / \mathrm{d} t$ in the current by $\langle H\rangle=\Gamma\left\langle\Delta f_{0} / f_{0}\right\rangle f_{0} \mathrm{~d} I /$ $\mathrm{d} f={ }_{1} \Gamma m / m_{0} f_{0} \mathrm{~d} I / \mathrm{d} f$, where $\mathrm{d} I / \mathrm{d} f$ is the slope of the resonance curve at the operating point, taken to be roughly constant over a significant portion of the side of a resonance. The shot noise power $S$ of $H$ is $S=2 \Gamma\left\langle\Delta I_{0}{ }^{2}\right\rangle$, where $\left\langle\Delta I_{0}{ }^{2}\right\rangle$ is the mean-square current increase from a single atom adsorption event. From this we find $t_{0} \sigma^{2}=3 /{ }_{4} m / m_{0} \mathrm{~d} I / \mathrm{d} f f_{0}\langle H\rangle$, where $t_{0}$ is an averaging time window $\left(1 / t_{0}\right.$ is the effective measurement bandwidth) and $\sigma$ is the standard deviation of the window-averaged $H$. This expression can be rearranged to express the mass ratio $\mathrm{m} / \mathrm{m}_{0}$ in terms of only known or measurable parameters:

$$
\frac{m}{m_{0}}=\frac{4}{3} \frac{t_{0} \sigma^{2}}{\langle H\rangle f_{0} \mathrm{~d} I / \mathrm{d} f}
$$

We now use this result to determine $m_{0}$ from the experimental data. The inset to Figure 4c shows a plot of $H$ with $t_{0}=50 \mathrm{~s}$ for the data shown in the main panel. Inspecting eq 2 indicates that if we plot an effective noise power $S_{\mathrm{N}}={ }_{4}^{4} t_{0} \sigma^{2}$ versus an effective mass current $I_{\text {mass }}=$ $\langle H\rangle f_{0} \mathrm{~d} I / \mathrm{d} f$, we expect the data to fall on a straight line with a slope equal to $m / m_{0}$. The solid circles in Figure $4 \mathrm{c}$ show $S_{\mathrm{N}}$ versus $I_{\text {mass }}$ taken from five different data sets. In each data set, to account for excess fluctuations due to the background noise, we subtract the variance taken from before mass loading from the variance taken during mass loading. Fitting a straight line that goes through the origin using statistical weighting (blue line) yields a slope $1.4 \times 10^{-4}$. Using the known mass of $\mathrm{Xe}$ of $0.218 \mathrm{zg}$ yields a nanotube mass $m_{0} \sim 1600 \mathrm{zg}$, consistent with the expected value based on the nanotube diameter and length. We have therefore used Xe to weigh the suspended nanotube segment.

With the measured mass of the nanotube, the atomic mass of Ar can then be determined from our data such as shown in the inset to Figure $4 d$. Figure $4 d$ shows $S_{\mathrm{N}}$ versus $I_{\text {mass }}$ data taken from four different data sets. Fitting a straight line through the origin to these points using statistical weighting (red line) yields a slope of $5.5 \times 10^{-5}$. Using the measured value for $m_{0}$ yields an Ar atomic mass of 0.085 $\mathrm{zg}$, in agreement to the expected mass of $0.066 \mathrm{zg}$. We have therefore weighed the potentially unknown atomic species of Ar using the mass-calibrated nanotube, demonstrating the potential of using nanotubes to perform ultraminiaturized mass spectrometry on a chip.

The atomic-scale mass resolution we observe here has significant scope for further improvement, for example, by using shorter nanotubes to reduce the nanotube mass, achieving better contacts, lowering the temperature to increase $\mathrm{d} G / \mathrm{d} V_{\mathrm{g}}$, and maintaining the drive frequency near resonance using feedback to improve the dynamic range of mass sensitivity. Such further improvement may conceivably permit a resolution on the order of the proton mass $\sim 1 \mathrm{yg}$, which could enable isotopic or chemical identification. Finally, for sufficiently short nanotubes, room temperature operation of the nanotubes as single-electron transistors ${ }^{26}$ may also yield highly sensitive detecting and weighing of adsorbates. 
Note Added in Proof: Following the submission of our manuscript, similar work by K. Jensen, K. Kim, and A. Zettl appeared in Nature Nanotech.

Acknowledgment. We thank C. N. Lau, D. H. Cobden, Z. H. Wang, O. Vilches, A. Bachtold, X. L. Feng, J. S. Gao, H. Maune, and I. Kozinsky for helpful discussions. We thank M. Gerfen for the cryostat construction. We thank P. Kelly, N. Asplund, S. Mitrovic, and C. Hughes for helpful discussions concerning the cryostat design. We thank the Micro/ Nano Fabrication Laboratory and the Kavli Nanoscience Institute at Caltech, the Center For Nanoscale Science and Engineering at UCR, and the Nanoelectronics Research Facility at UCLA where the sample fabrication was performed. M.B. acknowledges support by funding from NSFNRI, the Ross Brown Foundation, and ONR.

\section{References}

(1) Kong, J.; Franklin, N. R.; Zhou, C. W.; Chapline, M. G.; Peng, S.; Cho, K. J.; Dai, H. J. Science 2000, 287, 622-625.

(2) Lin, Y.; Taylor, S.; Li, H.; Fernando, K. A. S.; Qu, L.; Wang, W.; Gu, L.; Zhou, B.; Sun, Y.-P. J. Mater. Chem. 2004, 14, 527-541.

(3) Patolsky, F.; Lieber, C. M. Mater. Today 2005, 8, 20-28.

(4) Sidles, J. A.; Garbini, J. L.; Bruland, K. J.; Rugar, D.; Zuger, O.; Hoen, S.; Yannoni, C. S. Rev. Mod. Phys. 1995, 67, 249-265.

(5) Roukes, M. Sci. Am. 2001, 285, 48-57.

(6) Lavrik, N. V.; Datskos, P. G. Appl. Phys. Lett. 2003, 82, 2697-2699.

(7) Yang, Y. T.; Callegari, C.; Feng, X. L.; Ekinci, K. L.; Roukes, M. L. Nano Lett. 2006, 6, 583-586.

(8) Ekinci, K. L.; Huang, X. M. H.; Roukes, M. L. Appl. Phys. Lett. 2004, 84, 4469-4471.

(9) Poncharal, P.; Wang, Z. L.; Ugarte, D.; de Heer, W. A. Science 1999, $283,1513$.

(10) Peng, H. B.; Chang, C. W.; Aloni, S.; Yuzvinsky, T. D.; Zettl, A. Phys. Rev. Lett. 2006, 97, 087203.

(11) Reulet, B.; Kasumov, A. Y.; Kociak, M.; Deblock, R.; Khodos, I. I.; Gorbatov, Y. B.; Volkov, V. T.; Journet, C.; Bouchiat, H. Phys. Rev. Lett. 2000, 85, 2829-2832.
(12) Nishio, M.; Sawaya, S.; Akita, S.; Nakayama, Y. Appl. Phys. Lett. 2005, 86, 133111.

(13) Kong, J.; Soh, H. T.; Cassell, A. M.; Quate, C. F.; Dai, H. Nature (London) 1998, 395, 878-881.

(14) Jarillo-Herrero, P.; Kong, J.; van der Zant, H. S.; Dekker, C.; Kouwenhoven, L. P.; De Franceschi, S. Nature (London) 2005, 434, 484-488.

(15) Dekker, C. Phys. Today 1999, 52, 22-28.

(16) Kouwenhoven, L. P.; Marcus, C. M.; McEuen, P. L.; Tarucha, S.; Westervelt, R. M.; Wingreen, N. S. Electron Transport in Quantum Dots. NATO ASI Conf. Proc. 1997,

(17) Knobel, R. G.; Cleland, A. N. Nature (London) 2003, 424, 291-293.

(18) Sazonova, V.; Yaish, Y.; Üstünel, H.; Roundy, D.; Arias, T. A.; McEuen, P. L. Nature (London) 2004, 431, 284-287.

(19) Witkamp, B.; Poot, M.; van der Zant, H. S. J. Nano Lett. 2006, 6, 2904-2908.

(20) Purcell, S. T.; Vincent, P.; Journet, C.; Binh, V. T. Phys. Rev. Lett. 2002, 89, 276103.

(21) Üstünel, H.; Roundy, D.; Arias, T. Nano Lett. 2005, 5, 523-526.

(22) Garcia-Sanchez, D.; Paulo, A. S.; Esplandiu, M. J.; Perez-Murano, F.; Forró, L.; Aguasca, A.; Bachtold, A. Phys. Rev. Lett. 2007, 99, 085501.

(23) Postma, H. W. C.; Kozinsky, I.; Husain, A.; Roukes, M. Appl. Phys. Lett. 2005, 86, 223105.

(24) Rayleigh, J. W. S. The Theory of Sound, 2nd ed.; Dover Publications: New York, 1976; Vol. 1.

(25) Ekinci, K. L.; Yang, Y. T.; Roukes, M. L. J. Appl. Phys. 2004, 95, 2682-2689.

(26) Postma, H. W. C.; Teepen, T.; Yao, Z.; Grifoni, M.; Dekker, C. Science 2001, 293, 76-79.

(27) Bolton, K.; Rosen, A. Phys. Chem. Chem. Phys. 2002, 4, 4481-4488.

(28) Romero, H. E.; Bolton, K.; Rosen, A.; Eklund, P. C. Science 2005, 307, 89-93.

(29) Kuznetsova, A.; J. T.; Yates, J.; Liu, J.; Smalley, R. E. J. Chem. Phys. 2000, 112, 9590-9598.

(30) Cleland, A. N.; Roukes, M. L. J. Appl. Phys. 2002, 92, 2758-2769.

(31) Yong, Y.; Vig, J. IEEE Trans. Ultrason. Ferroelectr. Freq. Control 1989, 36, 452-458.

NL802181C 